\title{
Renal Autotransplant and Celiac Artery Bypass for Aneurysmal Degeneration Related to Neurofibromatosis Type 1
}

\author{
Natalie A. Drucker MD MS ${ }^{1}$, Maher F. Blaibel MD², Santosh Nagaraju MBBS ${ }^{1,3}$, S. Keisin \\ Wang $\mathrm{MD}^{4}$, William Goggins $\mathrm{MD}^{1,3}$, Andres Fajardo $\mathrm{MD}^{4}$ \\ ${ }^{1}$ Department of Surgery, Indiana University School of Medicine, Emerson Hall 545 Barnhill \\ Drive, Room 125, Indianapolis, IN 46202 \\ ${ }^{2}$ Department of Urology, Indiana University School of Medicine, 535 Barnhill Drive, Suite 150, \\ Indianapolis, IN 46202 \\ ${ }^{3}$ Division of Transplant Surgery, Department of Surgery, Indiana University School of \\ Medicine, Indianapolis, IN 46202 \\ ${ }^{4}$ Division of Vascular Surgery, Department of Surgery, Indiana University School of Medicine, \\ 1801 North Senate Boulevard MPC2-3500, Indianapolis, IN 46202
}

Corresponding Author: Natalie A. Drucker - Indiana University School of Medicine, Department of Surgery - 545 Barnhill Drive, Emerson Hall Rm 125, Indianapolis, IN 46202. ndrucker@iupui.edu, 954-579-1061

Funding: This research did not receive any specific grant from funding agencies in the public, commercial, or not-for-profit sectors.

Financial Disclosures: There are no financial relationships relevant to this article to disclose.

Conflicts of Interest: There are no conflicts of interest to disclose.

Author Contributions: ND, MB, SW, and SN drafted manuscript and performed critical revisions of manuscript. WG and AF performed study conception and design and provided critical revisions to the manuscript. All authors have approved the final submission.

This is the author's manuscript of the article published in final edited form as:

Drucker, N. A., Blaibel, M. F., Nagaraju, S., Wang, S. K., Goggins, W., \& Fajardo, A. (2019). Renal Autotransplant and Celiac Artery Bypass for Aneurysmal Degeneration Related to Neurofibromatosis Type 1. Vascular and Endovascular Surgery, 53(6), 497-500. https://doi.org/10.1177/1538574419846711 


\begin{abstract}
We present a case of an eighteen-year-old female with neurofibromatosis type 1 (NF1) who presented with abdominal pain and weight loss secondary to chronic mesenteric ischemia due to celiac axis occlusion and was subsequently found to have multiple visceral artery aneurysms. Of clinical significance, two aneurysms of the right renal artery were noted at the hilum with the larger one having a diameter of $2.4 \mathrm{~cm}$. After initial endovascular treatment with stenting of a concurrent pancreaticoduodenal artery pseudoaneurysm, staged aorto-hepatic bypass and right nephrectomy with renal auto-transplantation after back table resection of the aneurysmal segments was successfully completed.
\end{abstract}

Key words: neurofibromatosis, Von Recklinghausen's disease, renal artery aneurysm, autotransplantation, celiac artery bypass 
Neurofibromatosis type 1 (NF1), also known as von Recklinghausen disease, is an autosomal dominant condition with an uncommon, but well-established, association with peripheral vascular abnormalities, specifically aneurysms and stenoses [1]. We present a case of an eighteen-year-old female who developed celiac axis occlusion in conjunction with multiple visceral artery aneurysms including two saccular aneurysms of the right renal artery at the hilum. After endovascular stenting of a concurrent pancreaticoduodenal artery pseudoaneurysm, her postprandial pain slightly improved. She subsequently underwent concurrent aorto-hepatic bypass with right nephrectomy, back-table resection of the renal artery aneurysms, and autotransplantation into the right iliac fossa, which has not previously been described for NF1 vasculopathy. Informed consent has been obtained from the patient for publication of the case report and accompanying images.

Case Report: An 18-year-old female with a known history of NF1 presented to her primary care doctor with intermittent vague postprandial abdominal pain over the last six months. The pain was associated with nausea, occasional emesis, and crampy pain. She denied fever but did endorse some weight loss and food aversion. She reported normal and regular menstrual periods. Her vital signs were within normal limits and the physical exam was unremarkable without abdominal tenderness or masses. Routine blood laboratory evaluation also demonstrated normal renal and hepatic function.

Computed tomographic angiography (CTA) of the abdomen and pelvis demonstrated multiple vascular abnormalities including the following: (1) complete occlusion of the celiac artery at its origin, (2) retrograde filling of the hepatic and left gastric arteries from superior mesenteric artery (SMA) branches, (3) pancreaticoduodenal artery pseudoaneurysm, (4) focal $9 \mathrm{~mm}$ SMA ectasia, (5) patent inferior mesenteric artery (IMA) with a $6 \mathrm{~mm}$ focal ectasia, and (6) two saccular aneurysms of the right renal artery measuring approximately 1.0 and $2.4 \mathrm{~cm}$ in diameter, (7) $8 \mathrm{~mm}$ focal ectasia of the left renal artery.

Further evaluation of the right renal artery was performed with digital subtraction angiography (DSA) to further clarify the anatomy (Figure 1). Additionally, a 5 x $35 \mathrm{~mm}$ Pipeline stent (Medtronic, Minneapolis MN) was placed to protect the pseudoaneurysmal pancreaticoduodenal artery, the predominant collateral blood supply to the celiac distribution (Figure 2). This resulted in improvement of the patient's postprandial symptoms, and she was able to tolerate food without nausea or abdominal pain. However, over time her symptoms returned, and while we had initially planned to avoid a celiac bypass, we felt that it was necessary at this point. Additionally, her pre-operative CT demonstrated occlusion of the pancreaticoduodenal artery stent.

Therefore, three months after DSA and visceral stenting, she underwent a joint operation with transplant and vascular surgical teams. A right nephrectomy was performed through a midline laparotomy. The patient was heparinized prior to removal of the right kidney. The kidney was flushed on the back bench. Due to the anatomy of the aneurysms, the artery was divided distal to the aneurysms leaving the branches to the upper and lower poles separated but with normal caliber and appearance (Figure 3). The renal arteries were widely separated and too short to be reconstructed. While the transplant team performed the back-bench procedure, the vascular team performed a bypass from the supraceliac aorta to common hepatic artery with a reversed 
saphenous vein conduit. The right iliac system was dissected intraperitoneally. The kidney was then autotransplanted into the right iliac fossa with two separate arterial anastomoses. The superior renal artery was sewn into the right common iliac artery and the inferior renal artery was sewn into the right external iliac artery (Figure 4). The renal vein was sewn to the external iliac vein and the ureter to the bladder over a double-j stent as in a standard renal transplant.

The surgical pathology for the arterial aneurysms demonstrated disruption of the internal elastic lamina resulting in aneurysmal dilatation of the renal artery, neointimal thickening, and Schwann cell hyperplasia with myxoid degenerative changes. Post-operatively, the patient's creatinine and liver function tests were initially slightly elevated, but all returned to baseline previous to discharge. Her hospital course was complicated by a urinary tract infection treated successfully with antibiotics. She was discharged on post-operative day nine and progressed well at home. Her ureteral stent was removed three weeks after surgery, and she continues to do well as observed during her one-month post-operative visit.

\section{Discussion:}

NF1 is an autosomal dominant genetic disorder caused by mutations in the NF1 gene on chromosome 17. The incidence is about 1 in 3000 but only half of all cases are inherited [2]. The resultant pathogenesis observed in NF1 is an alteration in neurofibromin, a GTPaseactivating protein encoded by the mutated gene that is present in many tissues. While it is wellknown that NF1 is associated with neurofibromas, café-au-lait spots, Lisch nodules, and learning disabilities, the involvement of the vasculature is less often reported.

NF1 is associated with hypertension, congenital heart defects, and vasculopathy. Cardiovascular disease is a significant cause of death in NF1 patients, who have an average life-span 14 years shorter than expected in the general population [3]. Increased baseline blood pressure is most often essential hypertension, but pheochromocytoma and renal artery stenosis are also documented in this population [4]. Congenital heart disease occurs in a small proportion of NF1 patients and includes pulmonic stenosis, aortic coarctation, conotruncal defects, and other varieties [5].

NF1 vasculopathy, the focus of this case report, is an entity of unknown frequency due to the fact that most patient are asymptomatic. Vessels of any size may be affected, and typically multiple vessels are involved. Stenosis is the most commonly encountered lesion, followed by aneurysmal degeneration. The pathophysiology is not completely understood but proliferation of Schwann cells and smooth muscle nodules within the vessel walls has been noted [6]. The most common site of symptomatic vasculopathy is the renal arteries, and these patients present with hypertension due to stenosis [3]. Another common site for symptomatic lesions is in the cerebrovascular circulation, where ischemic or hemorrhagic stroke can occur. While there are not recommendations for screening asymptomatic patients, any child with known NF1 and a neurologic complaint should undergo MRI for further work-up for tumor or vascular anomaly [7, 8]. Our patient had previously undergone two brain MRIs without evidence of involvement of the cerebrovascular circulation.

Renal artery aneurysms are also described in NF1, though stenosis is about eight times more common according to a historical review by the Mayo Clinic group [1]. Renal artery aneurysms 
are often distal or intrarenal, making surgical repair difficult or impossible. Both open and endovascular techniques have been described for renovascular disease, and nephrectomy is reserved for difficult anatomic lesions. The lesion in our patient was not amenable to open bypass or endovascular stenting due to its location distal to the renal artery bifurcation. We did not simply perform nephrectomy due to concern that eventually her left renal artery would develop an aneurysm and a left nephrectomy may be necessary. She would then require dialysis or an allotransplant. In such a young patient, we felt strongly that renal mass and function should be preserved.. With a successful autotransplant of her right kidney, her lifetime risk of needing dialysis or an allotransplant has been almost eliminated. This is true even if her left renal artery develops an aneurysm and she requires a left nephrectomy in the future. Autotransplantation in this setting has not been previously described in the NF1 population.

Our patient was symptomatic secondary to chronic mesenteric ischemia. This has been described in very few case reports, and these are often due to external compression from neurofibromas [9] or SMA dysplasia [10]. The celiac axis is rarely stenosed or occluded secondary to NF1 vasculopathy, with only 1 lesion identified in a review article of 97 patients [1]. Additionally, SMA and IMA aneurysms are rarely described, both of which were present in this patient [11].

In the general population, renal artery aneurysms are typically considered for repair when they reach a size of $2.5 \mathrm{~cm}$ or become symptomatic. The risk of rupture is higher in pregnancy, so women of child-bearing age are typically advised to undergo repair regardless of size. For aneurysms in the hilum, nephrectomy with autotransplantation after arterial reconstruction is the procedure of choice, as bypass and endovascular stenting are impossible in this location [12]. Because NF1 vasculopathy is rare and data is sparse, it is recommended that patients be treated in accordance with the guidelines of the general population [3]. Therefore, it was certainly appropriate to treat this patient's renal artery aneurysms. By proceeding with reconstruction and autotransplantation, functional renal mass was able to be preserved.

NF1 and other non-atherosclerotic conditions are rare causes of celiac occlusion and chronic mesenteric ischemia, which typically occur in the elderly population [13]. This patient was able to partially compensate for her occlusion by developing retrograde flow through the SMA branches. After stenting of her pancreaticoduodenal artery, her symptoms did improve. However, celiac occlusion is associated with increased flow through the GDA and pancreatic arteries, which can contribute to worsening aneurysmal disease; therefore, celiac revascularization should be performed when possible.

In our practice, non-NF1 patients are typically followed with a CTA six weeks post-operatively, and then yearly with ultrasonography to evaluate for progression or recurrence of disease. Currently there is no recommendation for increased frequency of surveillance in patients with NF1. Additionally, she will require ongoing surveillance with yearly ultrasound or CTA to monitor the left renal artery for aneurysmal degeneration.

Our patient's vascular disease was discovered on a CT scan ordered for generalized abdominal pain secondary to mesenteric ischemia. Many NF1 patients never realize that they have NF1 vasculopathy secondary to the asymptomatic nature of this pathology. The current 
recommendations for screening are not different from the general population. However, practitioners who care for these patients should keep in mind the increased risk of vascular disease in these otherwise healthy-appearing, young individuals and keep NF1 vasculopathy on the differential diagnosis for common symptoms such as hypertension, abdominal pain, and headache. Further research into NF1 vasculopathy is required to further understand the pathophysiology and appropriate management of the disease process.

Conclusion: We present a case of an 18-year-old female with NF1 who presented with celiac occlusion causing postprandial pain and two large hilar renal artery aneurysms. After initial stenting of her pancreaticoduodenal pseudoaneurysm, she underwent a nephrectomy, aortohepatic bypass, and autotransplantation of her right kidney into the right iliac fossa. She recovered well and now maintains normal renal mass and function as well as improved visceral circulation.

\section{Figure Legends:}

Figure 1. Angiography demonstrating two saccular aneurysms in the right renal hilum. The larger one measures $2.4 \mathrm{~cm}$ and the smaller one is $1 \mathrm{~cm}$.

Figure 2. A. Angiography demonstrating a very large anterior inferior pancreaticoduodenal artery with a $1 \mathrm{~cm}$ aneurysm (arrow). Also shown are small fusiform aneurysmal dilations of the origin of the superior mesenteric artery and first jejunal branch (arrowheads). B. Pipeline stent successfully deployed and early flow stasis noted in target aneurysm (arrow).

Figure 3. Kidney on the back bench with aneurysmal dilations evident. Artery was divided distal to aneurysm (arrow) yielding two arterial branches of normal caliber. Vein was of adequate length (arrow head).

Figure 4. Kidney autotransplanted into right iliac fossa with two arterial branches (arrows) separately anastomosed to the right common and external iliac artery at the bifurcation (hypogastric artery indicated by arrowhead).

\section{References:}

[1] Oderich GS, Sullivan TM, Bower TC, Gloviczki P, Miller DV, Babovic-Vuksanovic D, et al. Vascular abnormalities in patients with neurofibromatosis syndrome type I: clinical spectrum, management, and results. J Vasc Surg 2007;46(3):475-84.

[2] Feldkamp MM, Gutmann DH, Guha A. Neurofibromatosis type 1: piecing the puzzle together. Can J Neurol Sci 1998;25(3):181-91.

[3] Friedman JM, Arbiser J, Epstein JA, Gutmann DH, Huot SJ, Lin AE, et al. Cardiovascular disease in neurofibromatosis 1: report of the NF1 Cardiovascular Task Force. Genet Med 2002;4(3):105-11.

[4] Ferner RE. The neurofibromatoses. Pract Neurol 2010;10(2):82-93.

[5] Lin AE, Birch PH, Korf BR, Tenconi R, Niimura M, Poyhonen M, et al. Cardiovascular malformations and other cardiovascular abnormalities in neurofibromatosis 1 . Am J Med Genet 2000;95(2):108-17.

[6] Huffman JL, Gahtan V, Bowers VD, Mills JL. Neurofibromatosis and arterial aneurysms. Am Surg 1996;62(4):311-4.

[7] Wolkenstein P, Freche B, Zeller J, Revuz J. Usefulness of screening investigations in neurofibromatosis type 1. A study of 152 patients. Arch Dermatol 1996;132(11):1333-6. 
[8] Lopez-Pison J, Cuadrado-Martin M, Boldova-Aguar MC, Munoz-Mellado A, Cabrerizo de Diago R, Pena-Segura JL. [Neurofibromatosis in children. Our experience]. Rev Neurol 2003;37(9):820-5.

[9] Cameron AJ, Pairolero PC, Stanson AW, Carpenter HA. Abdominal angina and neurofibromatosis. Mayo Clin Proc 1982;57(2):125-8.

[10] Cormier F, Cormier JM. [Thirty-eight cases of dysplasia of the superior mesenteric artery]. J Mal Vasc 2005;30(3):150-61.

[11] Hassen-Khodja R, Declemy S, Batt M, Castanet J, Perrin C, Ortonne JP, et al. Visceral artery aneurysms in Von Recklinghausen's neurofibromatosis. J Vasc Surg 1997;25(3):572-5.

[12] Lumsden AB, Salam TA, Walton KG. Renal artery aneurysm: a report of 28 cases. Cardiovasc Surg 1996;4(2):185-9.

[13] Schermerhorn ML, Giles KA, Hamdan AD, Wyers MC, Pomposelli FB. Mesenteric revascularization: management and outcomes in the United States, 1988-2006. J Vasc Surg 2009;50(2):341-8 e1. 


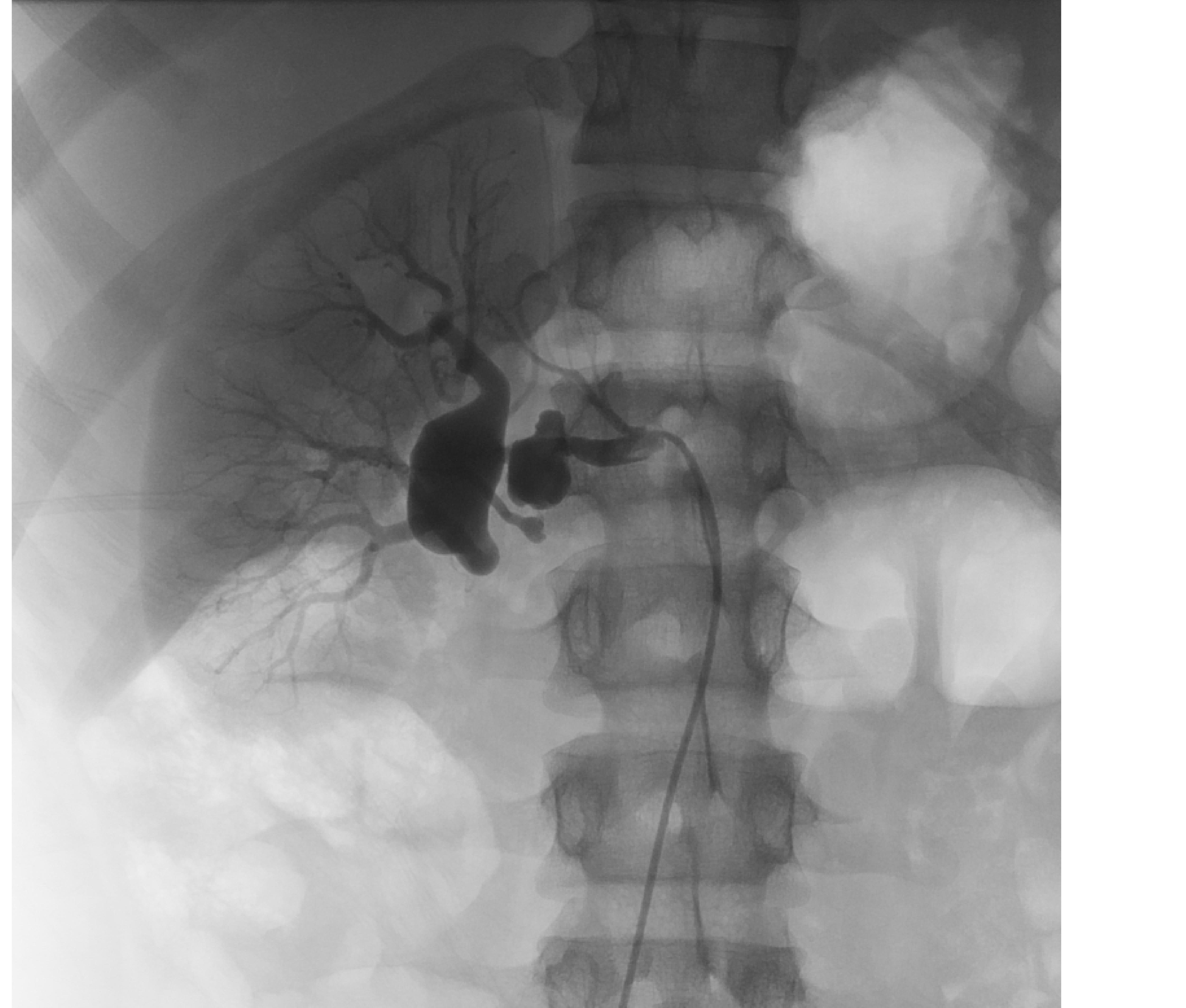




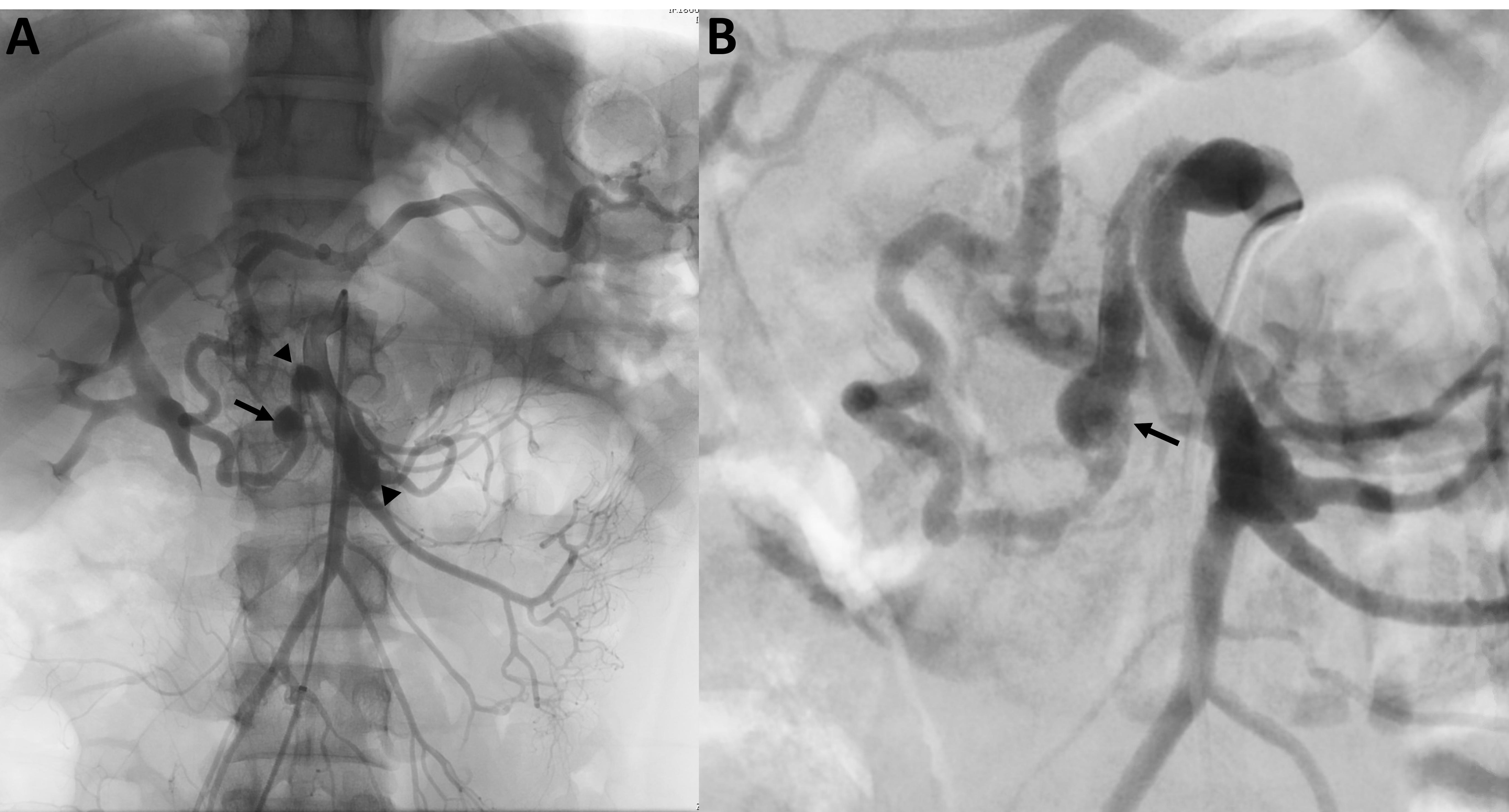





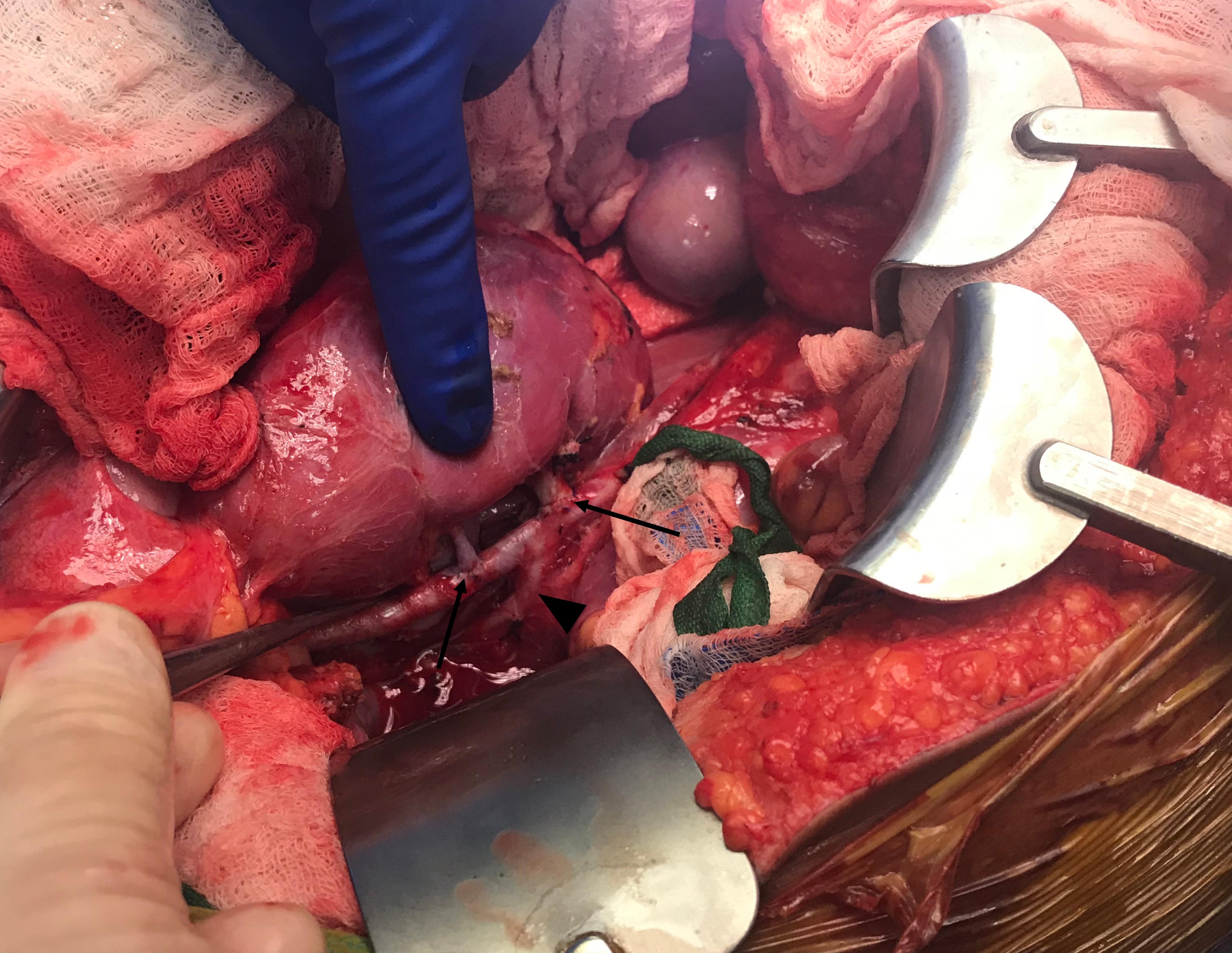


